\title{
NEW AND OLD FUNCTION SPACES IN THE THEORY OF PDES AND NONLINEAR ANALYSIS
}

\author{
TADEUSZ IWANIEC \\ Department of Mathematics, Syracuse University, Syracuse, NY 13244, U.S.A. \\ E-mail: tiwaniec@mailbox.syr.edu
}

CARLO SBORDONE

Dipartimento di Matematica ed Applicazioni "R. Caccioppoli", Università di Napoli Federico II via Cintia, 80126 Napoli, Italy

E-mail: sbordone@unina.it

1. Introduction. During the last twenty years, there has been a growing interest in the following questions:

- What is the natural domain of definition of a given differential equation?

- Which function spaces are best suited to the regularity of the solutions?

- How far beyond the natural domain we have the existence and uniqueness results?

- Can we detect analytic and geometric features of the solutions in these spaces?

These questions are due, to a large extent, to profound new developments in the geometric function theory [1], [45], calculus of variations [3], [20], [21], [34], [35] as well as some areas of applied mathematics [4], [61], [62]. An extensive use of new function spaces has been made in the setting of so-called very weak solutions of nonlinear PDEs [49] and mappings of finite distortion [44], [45]. Władysław Orlicz, a prominent Polish mathematician, did not perhaps suspect that the spaces he invented in 1932 [64], [2], [5], [6], [39], [54], [55], [56], [57], [65], [69], [72], [73], [74], [75] would enjoy such a high status today. A large part of this article is dedicated to a detailed exposition of the role of Orlicz spaces in modern approaches to nonlinear analysis, desirable and necessary. We shall in fact focus largely on topics related to our own research, though we mention a few contributions of a general nature. The aim is to illustrate some evidence of the necessity for using

2000 Mathematics Subject Classification: 35B65, 35F20, 35J70, 46E30, 46E35.

Research of T. Iwaniec was supported by NSF grants DMS-0301582 and DMS-024497.

Research of C. Sbordone was supported by GNAMPA-INdAM and the MIUR National Project "Variational Methods and Nonlinear Differential Equations".

The paper is in final form and no version of it will be published elsewhere. 
old and introducing new spaces of functions. We do not give the in-depth description of those topics, but we hope that there will be enough said to make it self-contained and interesting.

We are most grateful to the organizers of the meeting in Poznań. It was a special pleasure and honor for us to address a conference in the town of Władysław Orlicz.

2. Notation. The implied constant. A few brief comments on the notation used here are in order. Constants that arise in analysis are most often unconsidered constants. Whenever we see the inequality $\mathbb{X} \leqslant C \mathbb{Y}$ we tacitly understand that it holds with some positive constant $C$ independent of $\mathbb{X}$ and $\mathbb{Y}$. Having accepted such convention there is actually no need to use the letter $C$ at all. We therefore propose the abbreviation $\mathbb{X} \preccurlyeq \mathbb{Y}$. Sometimes, however, it will be desirable to know the implied constant (the one omitted from the inequality). Both symbols $\preccurlyeq$ and the reverse one $\succcurlyeq$ will give us not only a neat appearance to the estimates, that look awkward otherwise, but also will allow the implied constant to vary from line to line without confusion.

3. The Orlicz space $\mathscr{L}^{P}(\Omega)$. It will be necessary to have at hand a slightly more general definition than the usual ones.

An Orlicz function is any $\mathscr{C}^{\infty}$-smooth increasing function $P:[0, \infty) \rightarrow[0, \infty)$ such that $P(0)=0$ and $P(\infty)=\lim _{t \rightarrow \infty} P(t)=\infty$. Let $\Omega$ be an open subset of $\mathbb{R}^{n}$, or any $\sigma$-finite measure space. The Orlicz class $\mathscr{L}^{P}(\Omega)$ consists of measurable functions $f$ on $\Omega$ such that

$$
\|f\|_{P}=\|f\|_{\mathscr{L}^{P}(\Omega)} \stackrel{\text { def }}{=} \inf \left\{\frac{1}{\lambda}: \int_{\Omega} P(\lambda|f(x)|) \mathrm{d} x \leqslant 1\right\}
$$

is finite. If $P$ is convex we usually call it a Young function. In this case the Luxemburg functional \|\|$_{P}$ is a norm making $\mathscr{L}^{P}(\Omega)$ into a Banach space. In what follows we will be largely concerned with spaces very close to $\mathscr{L}^{1}(\Omega)$, that is, when $P$ exhibits a growth not far away from the identity function.

Definition 3.1. An Orlicz function $P$ is said to increase almost linearly if

$$
\lim _{t \rightarrow \infty} \frac{t P^{\prime}(t)}{P(t)}=1
$$

We should recall that the behavior of $P=P(t)$ on any finite interval will play no role in our discussion. However, we shall conveniently assume that $P(t)=O\left(t^{2}\right)$ at $t=0$. To every such $P$ there corresponds the so-called adjacent function. For the definition there are two cases to consider:

- $P$ satisfies the so-called divergence condition

$$
\int_{0}^{\infty} \frac{P(s) \mathrm{d} s}{s^{2}}=\infty .
$$

Then its adjacent function is defined by the rule

$$
P_{+}(t)=t \int_{0}^{t} \frac{P(s) \mathrm{d} s}{s^{2}} .
$$


- $P$ satisfies the convergence condition

$$
\int_{0}^{\infty} \frac{P(s) \mathrm{d} s}{s^{2}}<\infty
$$

Then its adjacent function is:

$$
P_{-}(t)=t \int_{t}^{\infty} \frac{P(s) \mathrm{d} s}{s^{2}} .
$$

Both $P_{+}$and $P_{-}$are Orlicz functions. A glance at the second derivatives shows that

$$
\frac{\mathrm{d}^{2} P_{ \pm}}{\mathrm{d} t^{2}}= \pm \frac{P^{\prime}(t)}{t}, \quad \text { respectively. }
$$

Thus $P_{+}$is always convex while $P_{-}$is always concave. Condition (1) just amounts to saying that the adjacent functions increase to infinity substantially faster than $P$, that is,

$$
\lambda(t) \stackrel{\text { def }}{=} \frac{P_{ \pm}(t)}{P(t)} \rightarrow \infty \quad \text { as } t \rightarrow \infty,
$$

For reasons to be clarified later on we refer to $\lambda$ as the improvement quotient. The importance of the adjacent Orlicz functions is borne out by several results concerning higher integrability of null Lagrangians, see Section 13. By way of digression, under the divergence condition we have the following inequalities [37], [51], [52]:

$$
\|\mathbf{M} f\|_{P} \preccurlyeq\|f\|_{P_{+}} \preccurlyeq\|\mathbf{M} f\|_{P}
$$

where $\mathbf{M}$ stands for the Hardy-Littlewood maximal operator, $\Omega$ being a cube in $\mathbb{R}^{n}$. The familiar case of E. Stein [71], [69] arises when $P(t)=\frac{t^{2}}{1+t}$, so $P_{+}(t)=t \log (1+t)$. More generally, if the maximal function of a Borel measure $\mu$ on $\Omega$ belongs to $\mathscr{L}^{P}(\Omega)$ then $\mu$ is absolutely continuous and its Radon-Nikodym derivative $\mu^{\prime}(x)$ is a function in the Orlicz space $\mathscr{L}^{P}(\Omega)$

$$
\left\|\mu^{\prime}\right\|_{P_{+}} \preccurlyeq\|\mathbf{M} \mu\|_{P} .
$$

Later we shall briefly discuss it in the context of Hardy-Orlicz spaces. Here are some pairs of adjacent Orlicz functions and the corresponding improvement quotients:

$$
\begin{array}{ll}
P_{ \pm} \approx t \exp ( \pm \sqrt{\log t}), & \lambda \approx \sqrt{\log t}, \\
P_{ \pm} \approx t(\log t)^{ \pm 1}, & \lambda \approx \log t, \\
P_{ \pm} \approx t(\log \log t)^{ \pm 1}, & \lambda \approx(\log t) \log \log t .
\end{array}
$$

Observe the pattern:

The closer $P_{ \pm}$is to the identity function, the larger improvement quotient appears.

The adjacent functions get arbitrarily close, but never reach, the identity function. In view of Condition (1) all the improvement quotients $\lambda(t)=\frac{P_{ \pm}(t)}{P(t)}$ have limited growth, controlled by the equation

$$
\int_{1}^{\infty} \frac{d t}{t \lambda(t)}=\infty
$$

Special Orlicz spaces will be discussed in the subsequent sections. 


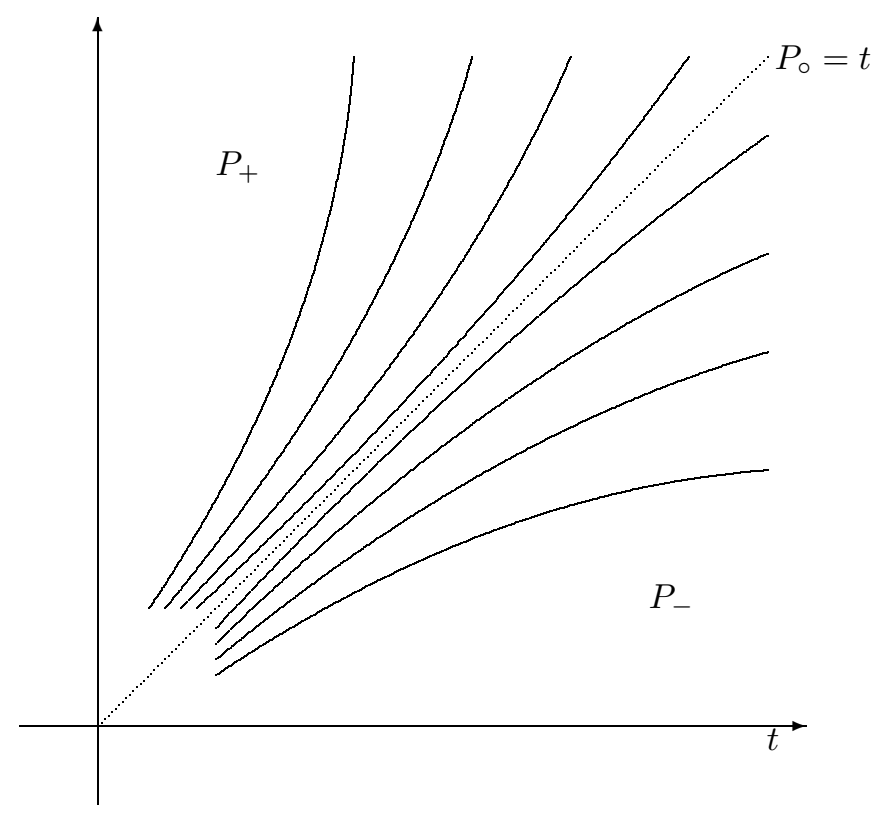

Fig. 1

4. The space $B M O(\Omega)$. At the other end of the scale of the functions to be discussed lies $B M O(\Omega)$ [53]. Let $\Omega$ be an open subset of $\mathbb{R}^{n}$. A function $u \in \mathscr{L}_{\text {loc }}^{1}(\Omega)$ is said to have bounded mean oscillations if

$$
\|u\|_{B M O(\Omega)}=\sup \left\{f_{Q}\left|u-u_{Q}\right|: Q \subset \Omega\right\}<\infty
$$

where the supremum runs over all cubes $Q$ (or balls) in $\Omega$. As usual the symbols $f_{Q}$ and $u_{Q}$ stand for the integral mean over the set $Q$. Both cubes and balls are well suited to various geometric constructions in $\mathbb{R}^{n}$. However there is a simple technical innovation that leads to dimension free results. To this effect one must work with uneven cubes, call them building blocks. By definition, a block is a Cartesian product of closed intervals, $\mathbb{B}=\mathbb{I}_{1} \times \mathbb{I}_{2} \times \ldots \times \mathbb{I}_{n}$, where $\left|\mathbb{I}_{k}\right| \leqslant 2\left|\mathbb{I}_{l}\right|$, for $k, l=1,2, \ldots, n$. These building blocks supersede quite effectively the traditionally used dyadic cubes. While on this matter, let us observe that bisecting along the largest edge breaks a block into two building blocks. To illustrate the utility of this concept we introduce the BMO-norm

$$
\|u\|_{\mathrm{BMO}(\Omega)}=\sup \left\{f_{\mathbb{B}}\left|u-u_{\mathbb{B}}\right|: \mathbb{B} \subset \Omega\right\}
$$

where in this case $\mathbb{B}$ are the building blocks in $\Omega$. Here is a particularly elegant variant of the celebrated John-Nirenberg Lemma, see [43].

LEMMA 4.1. Let $\Omega$ be a building block in $\mathbb{R}^{n}$ and $u \in B M O(\Omega)$. Then

$$
f_{\Omega} \exp \left(\frac{\left|u(x)-u_{\Omega}\right|}{7\|u\|_{\text {BMO }(\Omega)}}\right) \mathrm{d} x \leqslant 36 .
$$


To our knowledge such dimension free estimates were never published. Weighted global exponential integrability of $u \in B M O\left(\mathbb{R}^{n}\right)$ are also of interest to us. We forgo dimension free estimates in order to allow for other $B M O$-norms.

LEMMA 4.2. There exists a constant $N=N(n)>0$ such that

$$
\int_{\mathbb{R}^{n}}\left[\exp \left(\frac{\left|u(x)-u_{\mathbb{Q}}\right|}{N\|u\|_{B M O\left(\mathbb{R}^{n}\right)}}\right)-1\right] \frac{\mathrm{d} x}{1+|x|^{n+1}} \leqslant 1
$$

where $u_{\mathbb{Q}}$ stands for the integral mean of $u$ over the unit cube in $\mathbb{R}^{n}$.

Roughly speaking, $u$ behaves like $\log (e+|x|)$ near $\infty$ in an average sense. More precise statement requires introducing so-called decreasing weights. These are measures in $\mathbb{R}^{n}$ of the form $\mathrm{d} \mu=w(|x|) \mathrm{d} x$, where $w:[0, \infty) \rightarrow[0, \infty)$ can be any function decreasing to zero, fast enough so that $\int_{\mathbb{R}^{n}} \mathrm{~d} \mu=1$.

LEMMA 4.3. For every $p \geqslant 1$ we have

$$
\left\|\frac{u-u_{\mathbb{Q}}}{\log (e+|x|)}\right\|_{L^{p}\left(\mathbb{R}^{n}, d \mu\right)} \preccurlyeq p\|u\|_{B M O\left(\mathbb{R}^{n}\right)}
$$

We emphasize explicitly that the implied constant does not depend on the weight. One usually takes $p=1$ and $\mathrm{d} \mu=\frac{\log (e+|x|)}{1+|x|^{n+1}} \mathrm{~d} x$ to obtain

$$
\int_{\mathbb{R}^{n}} \frac{\left|u(x)-u_{\mathbb{Q}}\right|}{1+|x|^{n+1}} \mathrm{~d} x \preccurlyeq\|u\|_{B M O\left(\mathbb{R}^{n}\right)} .
$$

5. The exponential class $\operatorname{Exp}(\Omega)$. One major unfavorable feature of the space $B M O(\Omega)$ is that the point-wise inequality $|v(x)| \leqslant|u(x)|$ with $u \in B M O(\Omega)$ does not guarantee that $v$ too has bounded mean oscillations. By contrast, the Orlicz space $\operatorname{Exp}(\Omega)$ does have this dominated type property.

We say that a function $u$ belongs to the exponential class $\operatorname{Exp}(\Omega)$ if

$$
\|u\|_{\operatorname{Exp}(\Omega)}=\inf \left\{\frac{1}{\lambda}: \int_{\Omega} \frac{e^{\lambda|u(x)|}-1}{1+|x|^{n+1}} \mathrm{~d} x \leqslant 1\right\}
$$

is finite. This expression gives us an order preserving norm in $\operatorname{Exp}(\Omega)$. Lemma 4.2 tells us that

$$
\left\|u-u_{\mathbb{Q}}\right\|_{\operatorname{Exp}(\Omega)} \leqslant N\|u\|_{B M O\left(\mathbb{R}^{n}\right)}
$$

Although the exponentially integrable functions need not have bounded mean oscillations they can be majorized by such functions.

Proposition 5.1. Let $v \in \operatorname{Exp}(\Omega)$ on some measurable set $\Omega \subset \mathbb{R}^{n}$. Then there exists $u \in B M O\left(\mathbb{R}^{n}\right)$ such that

$$
\begin{gathered}
|v(x)| \leqslant u(x) \quad \text { almost everywhere in } \Omega, \\
\|u\|_{B M O\left(\mathbb{R}^{n}\right)} \leqslant c_{n}\|v\|_{\operatorname{Exp}(\Omega) .}
\end{gathered}
$$

Proof. It involves no loss of generality to assume that $\|v\|_{\operatorname{Exp}(\Omega)}=\frac{1}{2}$. Thus, by the definition of the exponential norm,

$$
\int_{\Omega} \frac{\left(e^{|v(x)|}-1\right) \mathrm{d} x}{1+|x|^{n+1}} \leqslant 1 .
$$


We then conveniently regard $v$ as a function defined on the entire space $\mathbb{R}^{n}$, say equal to zero outside $\Omega$. This gives us a function $\Phi(x)=\frac{e^{|v(x)|}}{1+|x|^{n+1}} \in \mathscr{L}^{1}\left(\mathbb{R}^{n}\right)$. We can now define the majorant of $v$ by the rule

$$
u(x)=(\log \mathbf{M} \Phi)(x)+\log \left(1+|x|^{n+1}\right) .
$$

Next we appeal to R. Coifman and R. Rochberg [18], who proved that $\log \mathbf{M} \Phi \in$ $B M O\left(\mathbb{R}^{n}\right)$. We also obtain from [18] the uniform bound

$$
\|u\|_{B M O\left(\mathbb{R}^{n}\right)} \leqslant \frac{1}{2} c_{n}=c_{n}\|v\|_{\operatorname{Exp}(\Omega)}
$$

where $c_{n}$ is a universal constant. For the inequality (18) we need only observe that

$$
u(x) \geqslant \log \Phi(x)+\log \left(1+|x|^{n+1}\right)=|v(x)|
$$

in $\Omega$, completing the proof of Proposition 5.1.

6. Nonisotropic degenerate elliptic PDE. One of the motivations for the study of $B M O$-majorization was to solve the Dirichlet problem [50]

$$
\left\{\begin{array}{l}
\operatorname{div} \mathbf{A}(x) \nabla u=\operatorname{div} \mathbf{A}(x) F, \\
u(x)=0 \quad \text { on } \partial \Omega
\end{array}\right.
$$

where $\mathbf{A}(x)$ is a symmetric matrix function with measurable entries satisfying the ellipticity condition

$$
K^{-1}(x)|\xi|^{2} \leqslant\langle\mathbf{A}(x) \xi, \xi\rangle \leqslant K(x)|\xi|^{2}, \quad \xi \in \mathbb{R}^{n},
$$

where $K \in \operatorname{Exp}(\Omega)$. The natural domain of definition for such equations is the Sobolev class of functions vanishing on $\partial \Omega$ and having finite energy

$$
\mathscr{E}[\nabla u]=\int_{\Omega}\langle\mathbf{A}(x) \nabla u, \nabla u\rangle \mathrm{d} x<\infty .
$$

If a given vector field $F=F(x)$ also has finite energy, meaning that $\langle\mathbf{A} F, F\rangle \in \mathscr{L}^{1}(\Omega)$, then the existence and uniqueness follow by variational arguments. In this natural setting we have the fundamental estimate $\mathscr{E}[\nabla u] \leqslant \mathscr{E}[F]$. Before jumping to a desirable conclusion we point out that by Hölder's inequality the assumption $K \in \operatorname{Exp}(\Omega)$ places the solution fields $\mathcal{E}=\nabla u$ and $\mathcal{B}=\mathbf{A}(\nabla u-F)$ in the Orlicz space $\mathscr{L}^{2} \log ^{-1} \mathscr{L}(\Omega)$, which is rather unsatisfactory. The finite energy assumption on $F$ is insufficient for the $\mathscr{L}^{2}$-conclusions. If, instead, we assume that $K F \in \mathscr{L}^{2}\left(\Omega, \mathbb{R}^{n}\right)$ and that $\|K\|_{\operatorname{Exp}(\Omega)}$ is rather small, then the $\mathscr{L}^{2}$-estimate takes the form

$$
\int_{\Omega}\left(|\mathcal{E}(x)|^{2}+|\mathcal{B}(x)|^{2}\right) \mathrm{d} x \preccurlyeq \int_{\Omega} K^{2}(x)|F(x)|^{2} \mathrm{~d} x .
$$

The proof is based on a celebrated result of harmonic analysis, the $\mathscr{H}^{1}-B M O$ duality. In brief outline, the equation gives us a point-wise inequality

$$
|\mathcal{E}(x)|^{2}+|\mathcal{B}(x)|^{2} \preccurlyeq K(x)\langle\mathcal{E}(x), \mathcal{B}(x)\rangle+K^{2}(x)|F(x)|^{2} .
$$


At this point the interested reader may consult [50] for far reaching extensions of this idea. We aim to give a meaning to the integral

$$
\int_{\Omega} K(x)\langle\mathcal{E}(x), \mathcal{B}(x)\rangle \mathrm{d} x .
$$

To this end, we observe that $(\mathcal{B}, \mathcal{E})$ is a div-curl couple: $\operatorname{div} \mathcal{B}=0$ and $\operatorname{curl} \mathcal{E}=0$. Therefore, the scalar product $\langle\mathcal{B}, \mathcal{E}\rangle$ lies in the Hardy space $\mathscr{H}^{1}(\Omega)$, as shown by Coifman, Lions, Meyer and Semmes [17]. On the other hand, $K$ is majorized by a function with small $B M O$-norm. Using $\mathscr{H}^{1}-B M O$ duality we arrive at the estimate (26).

The next two sections shed more light on these topics.

7. The Hardy space $\mathscr{H}^{1}(\Omega)$. Fix a nonnegative function $\Phi \in \mathscr{C}_{0}^{\infty}\left(\mathbb{R}^{n}\right)$ supported in the unit ball and having integral 1. It generates a one parameter family $\left\{\Phi_{t}\right\}_{t>0}$ of mollifiers $\Phi_{t}(x)=t^{-n} \Phi\left(t^{-1} x\right)$. To every Schwartz distribution $f \in \mathscr{D}^{\prime}(\Omega)$ there correspond smooth functions $f_{t}(x)=\left(f * \Phi_{t}\right)(x)$ defined on $\Omega_{t}=\{x \in \Omega: \operatorname{dist}(x, \partial \Omega)>t\}$. Now the maximal operator on $\mathscr{D}^{\prime}(\Omega)$ is defined by the rule

$$
(\mathfrak{M} f)(x)=\sup \left\{\left|f_{t}(x)\right|: 0<t<\operatorname{dist}(x, \partial \Omega)\right\} .
$$

We say that $f \in \mathscr{H}^{1}(\Omega)$ if its norm

is finite.

$$
\|f\|_{\mathscr{H}^{1}(\Omega)}=\int_{\Omega}(\mathfrak{M} f)(x) \mathrm{d} x
$$

8. Null Lagrangians. The Fundamental Theorem of Calculus tells us that

$$
\int_{a}^{b} f^{\prime}(x) \mathrm{d} x=\int_{a}^{b} g^{\prime}(x) \mathrm{d} x
$$

whenever two functions $f, g \in A C[a, b]$ coincide at the endpoints of the interval. One might ask which integral expressions enjoy the indentity such as this. Let us clear up this question with another example. Suppose $F=F(x, y)$ is a given function of class $\mathscr{C}^{1}([a, b] \times \mathbb{R})$ and $E(x, y, z)=\frac{\partial F}{\partial x}+z \frac{\partial F}{\partial y}$. We again find ourselves in a situation when the values of any function $f \in A C[a, b]$ inside the interval have no impact on the energy integral

$$
\mathcal{E}[f] \stackrel{\text { def }}{=} \int_{a}^{b} E\left(x, f, f^{\prime}\right) \mathrm{d} x=\int_{a}^{b} \frac{\mathrm{d}}{\mathrm{d} x} F(x, f) \mathrm{d} x=F(b, f(b))-F(a, f(a)) .
$$

In higher dimensions there are many examples of nonlinear partial differential expressions whose integral over any domain reduces to the boundary integration. Most familiar is the Jacobian determinant

$$
J(x, f)=\operatorname{det}\left[\frac{\partial f^{i}}{\partial x_{j}}\right]
$$

of a mapping $f=\left(f^{1}, \ldots, f^{n}\right): \Omega \rightarrow \mathbb{R}^{n}$. Stokes' formula yields integration over the boundary

$$
\begin{aligned}
\int_{\Omega} J(x, f) \mathrm{d} x & =\int_{\Omega} \mathrm{d} f^{1} \wedge \mathrm{d} f^{2} \wedge \ldots \wedge \mathrm{d} f^{n} \\
& =\int_{\Omega} \mathrm{d}\left(f^{1} \mathrm{~d} f^{2} \wedge \ldots \wedge \mathrm{d} f^{n}\right)=\int_{\partial \Omega} f^{1} \mathrm{~d} f^{2} \wedge \ldots \wedge \mathrm{d} f^{n} .
\end{aligned}
$$


While it is beyond the scope of this survey to provide details, we can at least indicate that more general wedge products of exact differential forms enjoy such integral identities. Among them is the wedge product $\mathrm{d} u \wedge \mathrm{d} v$ of exact differential forms of degree 1 and $n-1$, respectively. It is customary to identify $\mathrm{d} u \wedge \mathrm{d} v$ with a scalar product of the vector fields:

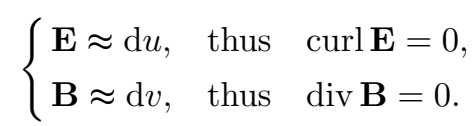

The concept of a div-curl couple owes much of its importance to Maxwell's equations. We call $\mathrm{d} u \wedge \mathrm{d} v=\langle\mathbf{E}, \mathbf{B}\rangle \mathrm{d} x$ the div-curl product.

With these preliminary examples we can broadly state that a nonlinear differential operator $\mathcal{N}(x, D)$ is called a null Lagrangian if

$$
\int_{\Omega} \mathcal{N}(x, D u) \mathrm{d} x=\int_{\Omega} \mathcal{N}(x, D v) \mathrm{d} x
$$

whenever $u=v$ on $\partial \Omega$. When discussing weakly differentiable Sobolev functions, this definition extends in the same guise as long as the integrals are meaningful. Mathematical principles of continuum mechanics, nonlinear elasticity theory and so forth grew out of the classical task to minimize the so-called stored energy functionals

$$
\mathcal{E}[u]=\int_{\Omega} E(x, D u) \mathrm{d} x
$$

subject to certain boundary conditions.

From this point of view, the null Lagrangians pertain to the integrands (nonlinear differential expressions) whose variational Lagrange-Euler equations are satisfied identically. The fundamentally analytic issue is the self improving regularity, a phenomenon that can be traced back at least to H. Wente (1969) and F. W. Gehring (1973), in relation with their studies of existence theorems for surfaces of constant mean curvature [76] and $L^{p}$-theory of quasiconformal mappings [30]. Today this subject has a life of its own [17], [60], [50], [36], [52], [48]. Null Lagrangians proved extremely useful in nonconvex calculus of variation. For example, the concept of polyconvex energy functionals originated from null Lagrangians [3].

9. $\mathscr{H}^{1}$-regularity of null Lagrangians. Let $f=\left(f^{1}, \ldots, f^{n}\right): \Omega \rightarrow \mathbb{R}^{n}$ be a mapping of Sobolev class $\mathscr{W}^{1, n}\left(\Omega, \mathbb{R}^{n}\right)$. We take on stage the Jacobian determinant

$$
J(x, f)=\operatorname{det} D f(x)=\operatorname{det}\left[\frac{\partial f^{i}}{\partial x_{j}}\right]
$$

as an example of null Lagrangians. The cofactors of the differential matrix $D f$ are also null Lagrangians. We accomodate all cofactors in a matrix denoted by $D^{\sharp} f$. Hadamard's inequality tells us that

$$
|J(x, f)| \leqslant\left|D^{\sharp} f(x)\right|^{\frac{n}{n-1}} \leqslant|D f(x)|^{n} .
$$

Proposition 9.1. We have

$$
\|\operatorname{det} D f\|_{\mathscr{H}^{1}(\Omega)} \preccurlyeq \int_{\Omega}\left|D^{\sharp} f(x)\right|^{\frac{n}{n-1}} \mathrm{~d} x \leqslant \int_{\Omega}|D(x)|^{n} \mathrm{~d} x .
$$


Proof. The first inequality holds if we assume that $f \in \mathscr{W}^{1, n-1}\left(\Omega, \mathbb{R}^{n}\right)$ and $\left|D^{\sharp} f\right| \in$ $\mathscr{L}^{\frac{n}{n-1}}(\Omega)$. There are two crucial ingredients in the proof of this inequality: the use of a weak form of isoperimetric inequality and a new maximal operator that combines both the Hardy-Littlewood and the spherical maximal operators.

It was the first time that the $\mathscr{L}^{p}$-theory $\left(p>\frac{n}{n-1}\right)$ of the spherical maximal operator [11], [70] was successfully exploited in the study of Jacobians. However, as this theory fails for $p=\frac{n}{n-1}$, we actually worked out the following operator. For each parameter $\theta \geqslant 1$ and $h \in \mathscr{L}^{p}\left(\mathbb{R}^{n}\right)$, we set

$$
\left(\mathbf{M}_{\theta} h\right)(x)=\sup _{t>0}\left[\frac{n}{t^{n}} \int_{0}^{t} r^{n-1}\left(\frac{1}{|S(x, r)|} \int_{S(x, r)}|h(y)| \mathrm{d} y\right)^{\theta} \mathrm{d} r\right]^{\frac{1}{\theta}} .
$$

The Hardy-Littlewood operator is none other than $\mathbf{M}_{1}$, while the spherical one arises by letting $\theta$ go to infinity. The operators $\mathbf{M}_{\theta}$ have the advantage of being bounded in $\mathscr{L}^{p}\left(\mathbb{R}^{n}\right)$ for all $p>\frac{n}{n-1+\frac{1}{\theta}}$. In particular, $\mathbf{M}_{\theta}$ is always bounded in $\mathscr{L}^{\frac{n}{n-1}}\left(\mathbb{R}^{n}\right)$. Unfortunately, we cannot put these beautiful arguments into play here [47]. Let us put more details on a somewhat weaker estimate

$$
\|\operatorname{det} D f\|_{\mathscr{H}^{1}(\Omega)} \preccurlyeq \int_{\Omega}|D f(x)|^{n} \mathrm{~d} x
$$

as these details will spark the forthcoming generalizations. To simplify matters we only consider mappings $f \in \mathscr{C}_{0}^{\infty}\left(\mathbb{R}^{n}, \mathbb{R}^{n}\right)$. Given a test function $\varphi \in \mathscr{C}_{0}^{\infty}\left(\mathbb{R}^{n}\right)$ we look at the differential 1-form $\varphi \mathrm{d} f^{1}$ and its Hodge decomposition into the exact and co-exact component

$$
\varphi \mathrm{d} f^{1}=\mathrm{d} \alpha+\mathrm{d}^{*} \beta .
$$

Stokes' theorem tells us that

$$
\int_{\mathbb{R}^{n}} \operatorname{det} D f=\int_{\mathbb{R}^{n}} \mathrm{~d} f^{1} \wedge \cdots \wedge \mathrm{d} f^{n}=\int_{\mathbb{R}^{n}} \mathrm{~d}\left(f^{1} \mathrm{~d} f^{2} \wedge \cdots \wedge \mathrm{d} f^{n}\right)=0 .
$$

By the same reasoning

$$
\int_{\mathbb{R}^{n}} \mathrm{~d} \alpha \wedge \mathrm{d} f^{2} \wedge \cdots \wedge \mathrm{d} f^{n}=0 .
$$

Therefore,

$$
\begin{aligned}
\int_{\mathbb{R}^{n}} \varphi(x) J(x, f) \mathrm{d} x & =\int_{\mathbb{R}^{n}} \varphi \mathrm{d} f^{1} \wedge \cdots \wedge \mathrm{d} f^{n}=\int_{\mathbb{R}^{n}}\left(\mathrm{~d}^{*} \beta\right) \wedge \mathrm{d} f^{2} \wedge \cdots \wedge \mathrm{d} f^{n} \\
& \leqslant \int\left|\mathrm{d}^{*} \beta\right| \cdot\left|\mathrm{d} f^{2}\right| \ldots\left|\mathrm{d} f^{n}\right| \leqslant\left\|\mathrm{d}^{*} \beta\right\|_{n}\|D f\|_{n}^{n-1} .
\end{aligned}
$$

Both exact and co-exact components can be expressed by means of a singular integral (Riesz transform) of $\varphi \mathrm{d} f^{1}$, say $\mathrm{d}^{*} \beta=\mathbf{T}\left(\varphi \mathrm{d} f^{1}\right)$. We rewrite it in the form of a commutator of $\mathbf{T}$ and the operator of multiplication by $\varphi$ :

$$
\mathrm{d}^{*} \beta=(\mathbf{T} \varphi-\varphi \mathbf{T}) \mathrm{d} f^{1} .
$$

This formula is justified since $\mathbf{T}$ vanishes on exact forms, by uniqueness in the Hodge decomposition. A point to emphasize here is that individually each term $\mathbf{T}(\varphi h)$ and $\varphi(\mathbf{T} h)$, for a general $h$, may not possess the desired regularity, but their difference does. 
As shown by Coifman, Rochberg and Weiss [19] the p-norms of the commutator

$$
\mathbf{T} \varphi-\varphi \mathbf{T}: \mathscr{L}^{p}\left(\mathbb{R}^{n}\right) \rightarrow \mathscr{L}^{p}\left(\mathbb{R}^{n}\right), \quad 1<p<\infty,
$$

are controlled linearly by the $B M O$-norm of the multiplier $\varphi$. In particular, we have

$$
\left\|d^{*} \beta\right\|_{n} \preccurlyeq\|\varphi\|_{B M O}\|D f\|_{n}
$$

and hence

$$
\int_{\mathbb{R}^{n}} \varphi(x) J(x, f) \mathrm{d} x \preccurlyeq\|\varphi\|_{B M O} \int_{\mathbb{R}^{n}}|D f(x)|^{n} \mathrm{~d} x
$$

This remains valid for mappings of Sobolev class $\mathscr{W}^{1, n}\left(\mathbb{R}^{n}, \mathbb{R}^{n}\right)$. Since $\mathscr{C}_{0}^{\infty}\left(\mathbb{R}^{n}\right)$ is dense in $V M O\left(\mathbb{R}^{n}\right)$, we see that $J(x, f)$ defines a bounded linear functional on $V M O\left(\mathbb{R}^{n}\right)$. As a last step we appeal to the $B M O-\mathscr{H}^{1}$ duality argument [23] to conclude with the desired estimate at (35).

10. The Rochberg-Weiss commutator. There are more commutators coming into play. As a starting point, we consider a singular integral operator $\mathbf{T}$ and the nonlinear operator

$$
\mathbf{T}^{\log }(h)=\mathbf{T}(h \log |h|)-(\mathbf{T} h) \log |\mathbf{T} h|
$$

defined for $h \in \mathscr{L}^{p}\left(\mathbb{R}^{n}\right)$, with $1<p<\infty$. Setting $\Phi=|h|+|\mathbf{T} h|$, we find the identity

$$
\mathbf{T}^{\log }(h)=\mathbf{T}(\varphi h)-\varphi \mathbf{T} h+\mathbf{T}\left(h \log \frac{|h|}{H}\right)-(\mathbf{T} h) \log \frac{|\mathbf{T} h|}{H}
$$

where $H=\mathbf{M} \Phi \geqslant|h|+|\mathbf{T} h|$ stands for the Hardy-Littlewood maximal function of $\Phi$, and $\varphi=\log \mathbf{M} \Phi \in B M O\left(\mathbb{R}^{n}\right)$. We then infer, in a rather straightforward way, that

$$
\left\|\mathbf{T}^{\log } h\right\|_{p} \preccurlyeq\|h\|_{p} .
$$

The above estimate is in effect a particular case of a general nonlinear interpolation theory of abstract bounded linear operators

$$
\mathbf{T}: \mathscr{L}^{s}(\mathbb{X}, \mu) \rightarrow \mathscr{L}^{s}(\mathbb{X}, \mu), \quad 1<s<\infty .
$$

We record the main estimate in the qualitatively best possible way, which accounts for a uniform bound of the modulus of continuity [51]

$$
\left\|\mathbf{T}^{\log } h-\mathbf{T}^{\log } f\right\|_{p} \preccurlyeq\|f-h\|_{p} \log \left(e+\frac{\|f\|_{p}+\|h\|_{p}}{\|f-h\|_{p}}\right) .
$$

There has since been a systematic study of nonlinear commutators. In this connection we mention Rochberg and Weiss studies of analytic families of Banach spaces [67] as well as N. Kalton's [54] and Milman-Rochberg's related works.

11. The $\mathscr{L} \log \mathscr{L}$-estimate. Just as in (36), we may decompose

$$
(\mathrm{d} f) \log |\mathrm{d} f|=\mathrm{d} \alpha+\mathrm{d}^{*} \beta,
$$

where $\mathrm{d} f=\left(\mathrm{d} f^{1}, \ldots, \mathrm{d} f^{n}\right), \mathrm{d} \alpha=\left(\mathrm{d} \alpha^{1}, \ldots, \mathrm{d} \alpha^{n}\right)$ and $\mathrm{d}^{*} \beta=\left(\mathrm{d}^{*} \beta^{1}, \ldots, \mathrm{d}^{*} \beta^{n}\right)$ are $n$ tuples of 1-forms. The co-exact component satisfies

$$
\left\|\mathrm{d}^{*} \beta\right\|_{n} \preccurlyeq\|\mathrm{d} f\|_{n}
$$


by the inequality (43). On substituting (45) into the inequality

$$
\int(\log |\mathrm{d} f|) \mathrm{d} f^{1} \wedge \mathrm{d} f^{2} \wedge \cdots \wedge \mathrm{d} f^{n}=\int \mathrm{d}^{*} \beta \wedge \mathrm{d} f^{2} \wedge \cdots \wedge \mathrm{d} f^{n} \leqslant\left\|\mathrm{~d}^{*} \beta\right\|_{n}\|D f\|_{n}^{n-1}
$$

we arrive [36] at a rather surprising estimate

$$
\int_{\mathbb{R}^{n}} J(x, f) \log |D f(x)| \mathrm{d} x \preccurlyeq \int_{\mathbb{R}^{n}}|D f(x)|^{n} \mathrm{~d} x .
$$

At first glance this estimate seems to lack homogeneity, but in fact it does not.

12. The space $\mathscr{L}^{p} \log \mathscr{L}(\Omega)$. Local variants of (46), first observed by S. Müller [60], are also available. A precise statement requires a brief introduction to the Zygmund classes $\mathscr{L}^{p} \log \mathscr{L}(\Omega)$.

Let $(\mathbb{X}, \mu)$ be a sigma finite measure space and $1 \leqslant p<\infty$. The Zygmund class $\mathscr{L}^{p} \log \mathscr{L}(\mathbb{X}) \subset \mathscr{L}^{p}(\mathbb{X})$ consists of functions $\Phi: \mathbb{X} \rightarrow \mathbb{R}$ such that

$$
\|\Phi\|_{\mathscr{L}^{p} \log \mathscr{L}}=\left[\int_{\mathbb{X}}|\Phi|^{p} \log \left(e+\frac{|\Phi|}{\|\Phi\|_{p}}\right)\right]^{\frac{1}{p}}<\infty .
$$

This expression defines a norm, though the triangle inequality is far from being obvious. The space dual to $\mathscr{L} \log \mathscr{L}(\mathbb{X})$ is $\operatorname{Exp}(\mathbb{X})$, while for $p>1$ the dual to $\mathscr{L}^{p} \log \mathscr{L}(\mathbb{X})$ is another Zygmund space $\mathscr{L}^{q} \log ^{1-q}(\mathbb{X}), p+q=p \cdot q$.

Suppose now that the Jacobian determinant of a Sobolev mapping $f \in \mathscr{W}^{1, n}\left(\Omega, \mathbb{R}^{n}\right)$ is nonnegative. In what follows we refer to Sobolev mappings with nonnegative Jacobian as orientation preserving mappings. Then for each relatively compact subset $\mathbb{X} \subset \Omega$ we have

$$
\|\operatorname{det} D f\|_{\mathscr{L} \log \mathscr{L}(\mathbb{X})} \preccurlyeq \int_{\Omega}|D f(x)|^{n} \mathrm{~d} x
$$

where the implied constant depends only on $n, \mathbb{X}$ and $\Omega$. This seemingly insignificant improvement of the degree of summability of the Jacobian turns out to be critical in many situations: existence of minima of nonconvex variational integrals [60], regularity of mappings with finite distortion [45], and so forth.

Actually, S. Müller, Qi Tang and B. Yan [62] established local $\mathscr{L} \log \mathscr{L}$-integrability of $J(x, f)$ with the only requirement being that the cofactors of the differential matrix belong to $\mathscr{L}^{\frac{n}{n-1}}(\Omega)$. This is also a consequence of our earlier $\mathscr{H}^{1}$-estimate at (34) when confronted with [69].

13. A study of null Lagrangians in Orlicz spaces. There are a number of important questions in modern geometric function theory concerning nonnegative Jacobians (orientation preserving mappings) and other null Lagrangians. Here we shall focus on self improving integrability. Let us reveal in advance that this phenomenon can be observed only in the Orlicz classes that are sufficiently close to $\mathscr{L}^{1}$, as described in Definition 3.1. We have already mentioned the $\mathscr{L} \log \mathscr{L}$-integrability of the Jacobians of orientation preserving mappings in the Sobolev classes $\mathscr{W}^{1, n}\left(\Omega, \mathbb{R}^{n}\right)$. A somewhat dual question is concerned with the $\mathscr{L}^{1}$-integrability under minimal assumptions on the differential matrix. The first stepping-stones were made in [48]. For each relatively compact subset 
$\mathbb{X} \subset \Omega$ we have

$$
\int_{\mathbb{X}} J(x, f) \mathrm{d} x \leqslant C_{n}(\mathbb{X}, \Omega) \int_{\mathbb{X}} \frac{|D f(x)|^{n} \mathrm{~d} x}{\log \left(e+\frac{|D f(x)|}{|D f| \mathbb{X}}\right)}
$$

In other words, the condition $|D f|^{n} \in \mathscr{L}^{P}(\Omega)$, where $P(t) \approx t \log ^{-1}(t)$, implies local integrability of the Jacobian. At this point it is worth mentioning that the Jacobian also obeys the rule of integration by parts. Precisely it means that

$$
\int_{\Omega} \phi(x) J(x, f) \mathrm{d} x=-\int_{\Omega} f^{1} \mathrm{~d} \phi \wedge \mathrm{d} f^{2} \wedge \cdots \wedge \mathrm{d} f^{n}
$$

for every test function $\phi \in \mathscr{C}_{0}^{\infty}(\Omega)$. The same result holds if we only assume that the cofactors of the differential matrix $D f$ belong to $\mathscr{L}^{\frac{n}{n-1}} \log ^{-1} \mathscr{L}$, that is, $\left|D^{\sharp} f\right|^{\frac{n}{n-1}} \in$ $\mathscr{L}^{P}(\Omega)$, see [31].

A device needed to carry through these estimates is the isoperimetric inequality on cubes carefully crafted from Whitney's decomposition of the level sets $\{x \in \Omega$ : $\left.\left(\mathbf{M} D^{\sharp} f\right)(x)>t\right\}$. As a matter of fact the only assumption needed for local integrability of the Jacobian is that $P$ satisfies the divergence condition (2). Some additional, usually minor, assumptions on $P$ are in order, but we need not bother about it here. The general rule reads:

$$
\left.\underset{\left|D^{\sharp} f\right|^{\frac{n}{n-1}}}{\operatorname{or}}\right\} \in \mathscr{L}^{P}(\Omega) \quad \text { implies } \quad \operatorname{det} D f \in \mathscr{L}_{\mathrm{loc}}^{P_{+}}(\Omega)
$$

where $P_{+}$denotes, as in (3), the convex adjacent to $P$. This improvement of integrability is sharp. Note, in particular, that the condition $|D f|^{n} \in \mathscr{L} \log ^{-1} \mathscr{L}$ implies a slightly better result than (49), namely $\operatorname{det} D f \in \mathscr{L} \log \log \mathscr{L}$, see G. Moscariello [59].

If we move into the class of Orlicz spaces $\mathscr{L}^{P}(\Omega)$ with $P$ satisfying the convergence condition (4), then the Jacobian determinant is no longer integrable. However, we still observe a phenomenon of higher degree of summability as long as $P$ exhibits a nearly linear growth, see Condition (1). As before;

$$
|D f|^{n} \in \mathscr{L}^{P}(\Omega) \quad \text { implies } \quad \operatorname{det} D f \in \mathscr{L}_{\mathrm{loc}}^{P_{-}}(\Omega)
$$

We then reiterate the principle of Section 3 in the language of null Lagrangians.

\section{The highest improvement of regularity of null Lagragians takes place near $\mathscr{L}^{1}(\Omega)$.}

14. The power type commutator. The motivational philosophy behind the nonlinear commutators is to achieve some sort of cancellation of terms. For instance, in view of inequality (43), the logarithmic terms in the commutator

$$
\mathbf{T}^{\log } h=\mathbf{T}(h \log |h|)-(\mathbf{T} h) \log |\mathbf{T} h|
$$

turned out to be harmless for the estimates in Section 11. There are also possible other types of cancellations in nonlinear commutators that can be effectively exploited for the regularity theory of null Lagrangians. Let $\mathbf{T}: \mathscr{L}^{s}(\mathbb{X}, \mu) \rightarrow \mathscr{L}^{s}(\mathbb{X}, \mu), 1<s<\infty$, be a bounded linear operator. The object of our discussion is the commutator of $\mathbf{T}$ and the 
power function $h \mapsto|h|^{z} h$, where $h \in \mathscr{L}^{p}(\mathbb{X}, \mu)$ and $z$ is a complex parameter in the half plane $\Re e z>\frac{1}{p}-1$. It is trivial that the nonlinear operator

$$
\mathbf{T}^{z} h \stackrel{\text { def }}{=} \mathbf{T}\left(|h|^{z} h\right)-|\mathbf{T} h|^{z} \mathbf{T} h
$$

is bounded from $\mathscr{L}^{p}(\mathbb{X}, \mu)$ to $\mathscr{L}^{\frac{p}{1+\Re e z}}(\mathbb{X}, \mu)$. Moreover

$$
\left\|\mathbf{T}^{z} h\right\|_{\frac{p}{1+\Re e z}} \leqslant C_{p}(z)\|h\|_{p}^{1+\Re e z}
$$

Although the constant $C=C(z)$ vanishes when $z=0$, it is still far from being obvious that $C(z)$ is in fact small near the origin. Broadly speaking, the function $z \mapsto \mathbf{T}^{z} h$ being analytic in $z$ satisfies the Schwarz lemma. In this way we strengthen the estimate to the following [49] :

$$
\left\|\mathbf{T}^{z} h\right\|_{\frac{p}{1+\Re e z}} \leqslant C_{p} \cdot|z| \cdot\|h\|_{p}^{1+\Re e z}
$$

for all $z$ sufficiently small, say $|z| \leqslant \frac{p-1}{2 p}$. We should realize, however, that this estimate is by no means a trivial consequence of the Schwarz lemma. As an application, consider the Hodge decomposition

$$
|\mathrm{d} f|^{-\epsilon} \mathrm{d} f=\mathrm{d} \alpha+\mathrm{d}^{*} \beta
$$

for a Sobolev mapping $f \in \mathscr{W}^{1, n-\epsilon}\left(\mathbb{R}^{n}, \mathbb{R}^{n}\right), \epsilon$ being a small positive number. By arguments similar to those in $(46)$ we obtain

$$
\int_{\mathbb{R}^{n}}|D f(x)|^{-\epsilon} J(x, f) \mathrm{d} x \preccurlyeq \epsilon \int_{\mathbb{R}^{n}}|D f(x)|^{n-\epsilon} \mathrm{d} x .
$$

The local variants of this inequality are just as easy to deduce. For example, let $f: \Omega \rightarrow$ $\mathbb{R}^{n}$ be an orientation preserving map of Sobolev class $\mathscr{W}^{1, n-\epsilon}\left(\Omega, \mathbb{R}^{n}\right)$. Then for every pair of concentric balls $\mathbb{B} \subset 2 \mathbb{B} \subset \Omega$ we have

$$
f_{\mathbb{B}} \frac{J(x, f) \mathrm{d} x}{|D f(x)|^{\epsilon}} \preccurlyeq\left(f_{2 \mathbb{B}}|D f|^{\frac{n^{2}}{n+1}}\right)^{\frac{(n-\epsilon)(n+1)}{n^{2}}}+\epsilon f_{2 \mathbb{B}}|D f|^{n-\epsilon}
$$

One is tempted to pass to the limit as $\epsilon \rightarrow 0$ but that is illegitimate in general. We must assume here that the latter term converges to zero or at least stays bounded. In this way we are naturally led to an investigation of the so-called grand space $\mathscr{L}^{n)}(\Omega)$.

15. Grand spaces $\mathscr{L}^{p)}(\Omega)$. Let $(\mathbb{X}, \mu)$ be a finite measure space. For $1<p<\infty$ we shall consider functions on $\mathbb{X}$ which are integrable with every power $s<p$. The grand space $\mathscr{L}^{p)}(\mathbb{X})$ consists of those functions $f \in \bigcap_{0<s<p} \mathscr{L}^{s}(\mathbb{X})$ for which

$$
\|f\|_{p)}=\sup _{0<\epsilon \leqslant p-1}\left(\epsilon \int_{\mathbb{X}}|f|^{p-\epsilon}\right)^{\frac{1}{p-\epsilon}}<\infty .
$$

This makes $\mathscr{L}^{p)}(\mathbb{X})$ into a Banach space. The restriction on $\epsilon$ is immaterial, different restrictions yield equivalent norms. In the similar fashion we define $\mathscr{L}^{1)}(\Omega)$ as a metric space. Note that $\mathscr{L}^{p}(\mathbb{X})$ is not dense in the grand space $\mathscr{L}^{p)}(\mathbb{X})$. Its closure, denoted by $\mathscr{L}_{0}^{p)}(\mathbb{X})$, consists of functions satisfying

$$
\lim _{\epsilon \rightarrow 0} \epsilon \int_{\mathbb{X}}|f|^{p-\epsilon}=0
$$


To illustrate, let us take on stage the Marcinkiewicz class $\mathscr{L}_{\text {weak }}^{p}(\mathbb{X})$, which is contained in $\mathscr{L}^{p)}(\mathbb{X})$ but not in $\mathscr{L}_{0}^{p)}(\mathbb{X})$. The Orlicz class $\mathscr{L}^{p} \log ^{-1} \mathscr{L}(\mathbb{X})$, on the other hand, is contained in $\mathscr{L}_{0}^{p}(\mathbb{X})$.

Returning to our estimate at (58) we conclude that orientation preserving mappings $f: \Omega \rightarrow \mathbb{R}^{n}$ in the grand Sobolev space $\mathscr{W}^{1, n)}\left(\Omega, \mathbb{R}^{n}\right)$ have locally integrable Jacobian. There is one interesting example to look at: $f(x)=x /|x|$ in the unit ball $\mathbb{B} \subset \mathbb{R}^{n}$. The norm of the differential matrix $|D f(x)|=1 /|x|$ lies in $\mathscr{L}_{\text {weak }}^{n}(\mathbb{B}) \subset \mathscr{L}^{n)}(\mathbb{B})$, but not in $\mathscr{L}_{0}^{n)}(\mathbb{B})$. Its Jacobian determinant vanishes almost everywhere and, by trivial means, belongs to $\mathscr{L}_{\text {loc }}^{1}(\mathbb{B})$. However, the formula of integration by parts at $(50)$ fails. This anomaly is due to the fact that the right hand side of (50) represents a Schwartz distribution supported at the origin, the Dirac mass. It is the condition $|D f| \in \mathscr{L}_{0}^{n)}(\Omega)$ that guarantees integration by parts.

16. Measure in the right hand side. Applications of grand spaces $\mathscr{L}^{p)}(\Omega)$ and $\mathscr{L}_{0}^{p)}(\Omega)$ are much wider than we just discussed, see [24], [26], [28], [27], [38]. We add to this discussion one result concerning the nonlinear $n$-harmonic equation

$$
\begin{cases}\operatorname{div}|\nabla u|^{n-2} \nabla u= & \mu, \\ u=0 & \text { on } \partial \Omega,\end{cases}
$$

where $\mu$ is a signed Radon measure [38], [50]. This Dirichlet problem admits exactly one solution $u \in \mathscr{W}_{0}^{1, n)}(\Omega)$, meaning that

$$
\varepsilon \int_{\Omega}|\nabla u(x)|^{n-\varepsilon} \mathrm{d} x \preccurlyeq \mu(\Omega)
$$

for all $0<\varepsilon \leqslant n-1$. Furthermore, if $\mu$ is absolutely continuous then $|\nabla u| \in \mathscr{L}_{0}^{n)}(\Omega)$, that is,

$$
\lim _{\varepsilon \rightarrow 0} \varepsilon \int_{\Omega}|\nabla u|^{n-\varepsilon}=0
$$

17. The Hardy-Orlicz space $\mathscr{H}^{P}(\Omega)$. A distribution $f \in \mathscr{D}^{\prime}(\Omega)$ is said to be of Hardy-Orlicz class $\mathscr{H}^{P}(\Omega)$ if its maximal function $\mathfrak{M} f$ lies in $\mathscr{L}^{P}(\Omega)$. We write

$$
\|f\|_{\mathscr{H}^{P}(\Omega)} \stackrel{\text { def }}{=}\|\mathfrak{M} f\|_{\mathscr{L}^{P}(\Omega)}
$$

Of course the only interesting cases occur when $P$ is nearly linear in the sense of Definition 3.1. It is instructive to analyze a few basic results. In [51] it was shown that the divergence condition at (2) is necessary and sufficient for all positive distributions $f \in \mathscr{H}^{P}(\Omega)$ to be represented by locally integrable functions. Furthermore, for each relatively compact subset $\mathbb{X} \subset \Omega$ we have

$$
\|f\|_{\mathscr{L}^{P^{+}(\mathbb{X})}} \preccurlyeq\|f\|_{\mathscr{H}^{P}(\Omega)}
$$

To some degree the converse also holds, namely $\mathscr{L}^{P_{+}}(\mathbb{X}) \subset \mathscr{H}_{\text {loc }}^{P}(\Omega)$. A natural question arises whether all distributions in $\mathscr{H}^{P}(\Omega)$, regardless of their sign, are the Radon measures. This is the case for $\mathscr{H}^{1}(\Omega)$, and any other case when $P$ is convex. In the concave case the answer is in the negative [52]. In fact each space $\mathscr{H}^{P}(\Omega)$ contains a distribution which is not a measure whenever $P(t)=o(t)$ at infinity. 
One important discovery that brought new life to Hardy-Orlicz spaces is the regularity phenomenon of null Lagrangians. In this category of interesting results we include the following guideline rule for higher regularity:

The Jacobian determinant of a Sobolev mapping with $|D f|^{n} \in \mathscr{L}^{P}(\Omega)$

belongs to the Hardy-Orlicz space $\mathscr{H}_{\mathrm{loc}}^{P}(\Omega)$.

This principle has been confirmed in a number of cases [52].

18. The grand Hardy space $\mathscr{H}^{1)}(\Omega)$. We finally push one step further the requirement about the integrability of $\mathfrak{M} f$. A distribution $f \in \mathscr{D}^{\prime}(\Omega)$ belongs to the grand Hardy space $\mathscr{H}^{1)}(\Omega)$ if its maximal function lies in $\mathscr{L}^{1)}(\Omega)$, that is,

$$
\varepsilon \int_{\Omega}|\mathfrak{M} f(x)|^{1-\varepsilon} \mathrm{d} x=O(1) \quad \text { as } \varepsilon \rightarrow 0 .
$$

As an example, the Dirac mass at a point in $\Omega$ is a distribution in $\mathscr{H}^{1)}(\Omega)$. Its maximal function lies in the Marcinkiewicz class $\mathscr{L}_{\text {weak }}^{1}(\Omega) \subset \mathscr{L}^{1)}(\Omega)$. This example persuades us to introduce the weak Hardy space $\mathscr{H}_{\text {weak }}^{1}(\Omega)$ as well. Accordingly,

$$
\mathscr{H}_{\text {weak }}^{1}(\Omega) \subset \mathscr{H}^{1)}(\Omega) \text {. }
$$

Up to this point we have only looked at how function spaces facilitate understanding PDEs and some nonlinear differential forms, Jacobians and div-curl products. There is also reverse interaction. It is hard to resist the temptation to round out our survey with an example of such interaction. In a recent project [10] we exploited div-curl products as generators of $\mathscr{H}^{1)}(\Omega)$ to investigate the function $\mathfrak{b} \cdot \mathfrak{h}$, where $\mathfrak{b} \in B M O(\Omega)$ and $\mathfrak{h} \in \mathscr{H}^{1}(\Omega)$. In general, $\mathfrak{b} \cdot \mathfrak{h}$ need not be locally integrable. Nevertheless, by virtue of the $\mathscr{H}^{1}-B M O$ pairing we can give a meaning to this function as a Schwartz distribution. It operates on a test function $\phi \in \mathscr{C}_{0}^{\infty}(\Omega)$ by the rule: $(\mathfrak{b} \cdot \mathfrak{h})[\phi]=(\phi \mathfrak{b})[\mathfrak{h}]$, where we view the $B M O$-function $\phi \mathfrak{b}$ as a linear functional on $\mathscr{H}^{1}(\Omega)$. Among the many interesting properties of the distribution $\mathfrak{b} \cdot \mathfrak{h}$ we found that it lies in the grand Hardy space $\mathscr{H}^{1)}(\Omega)$. Let $\Omega$ be a cube. Then we also have a uniform bound

$$
\|\mathfrak{b} \cdot \mathfrak{h}\|_{\mathscr{H}^{1)}(\Omega)} \preccurlyeq\|\mathfrak{b}\|_{\mathrm{BMO}(\Omega)}\|\mathfrak{h}\|_{\mathscr{H}^{1}(\Omega)}
$$

provided the integral mean of $\mathfrak{b}$ is equal to zero. Actually, a slightly better result holds. The $B M O-\mathscr{H}^{1}$ product admits a decomposition

$$
\mathfrak{b} \cdot \mathfrak{h}=g+f
$$

where $g \in \mathscr{L}^{1}(\Omega)$ and $f$ is a distribution in the Hardy-Orlicz space $\mathscr{H}^{P}(\Omega)$,

$$
P(t)=t \log ^{-1}(e+t) \text {. }
$$

Let us end this section by noticing that both $\mathscr{L}^{1}(\Omega)$ and $\mathscr{H}^{P}(\Omega)$ are contained in the grand Hardy space. In symbols

$$
B M O \cdot \mathscr{H}^{1}=\mathscr{L}^{1}+\mathscr{H}^{P} \subset \mathscr{H}^{1)} .
$$

19. Mappings between manifolds. Sobolev mappings between Riemannian manifolds have come into widespread usage in modern geometry [12] and topology [77], [7], [22], [40], [14]. We have recently recounted in [41] some nuances of mappings $f: \mathbb{X} \rightarrow \mathbb{Y}$ 
between smooth compact oriented Riemannian manifolds, $\operatorname{dim} \mathbb{X}=n \geqslant 2$ and $\operatorname{dim}$ $\mathbb{Y}=m \geqslant 2$. First, it is highly desirable to know whether smooth mappings $f \in \mathscr{C}^{\infty}(\mathbb{X}, \mathbb{Y})$ are dense in the metric topology of a Sobolev class $\mathscr{W}^{1, p}(\mathbb{X}, \mathbb{Y})$. The collapse of the smooth approximation for the exponents below the dimension of $\mathbb{X}, p<n$, lies fairly deep in the concept of the topological degree [68]. After several attempts [8] this situation was finally settled by F. Hang and F. Lin [42], but their result required certain topological assumptions on both the domain and the target manifold. Despite this peculiarity, it is still possible to relax all those topological constraints for mappings of the Orlicz-Sobolev class $\mathscr{W}^{1, P}(\mathbb{X}, \mathbb{Y})$ slightly below $\mathscr{W}^{1, n}(\mathbb{X}, \mathbb{Y})$. These results are necessary if one wants to build the theory of deformations with unbounded distortion on manifolds. Let us illustrate the Marcinkiewicz-Sobolev class of mappings whose tangent bundle map $D f: \mathbf{T X} \rightarrow \mathbf{T} \mathbb{Y}$ lies in $\mathscr{L}_{\text {weak }}^{n}(\mathbb{X})$, that is, $|\{x \in \mathbb{X}:|D f(x)|>t\}|=O\left(t^{-n}\right)$.

ThEOREM 19.1. The closure of $\mathscr{C}^{\infty}(\mathbb{X}, \mathbb{Y})$ in the metric topology of the MarcinkiewiczSobolev space $\mathscr{W}_{\text {weak }}^{1, n}(\mathbb{X}, \mathbb{Y})$ consists of mappings such that

$$
|\{x \in \mathbb{X}:|D f(x)|>t\}|=o\left(t^{-n}\right) \quad \text { as } t \rightarrow \infty .
$$

The arguments establishing smooth approximation in a manifold setting are really different than one might a priori expect. These ideas, which involve so-called web like structure on $\mathbb{X}$, also work with great effectiveness for mappings in the Orlicz-Sobolev classes $\mathscr{W}^{1, Q}(\mathbb{X}, \mathbb{Y})$, with $Q(t)=P\left(t^{n}\right)$. Here the defining function $P$ must satisfy, among other technical conditions, the divergence condition (2). For example:

$$
Q(t)=\frac{t^{n}}{\ln (1+t) \ln \ln (e+t) \cdots \ln \ln \ldots \ln (e \cdot+t)} .
$$

We again see how the divergence condition (2) plays a crucial role in geometric analysis.

TheOREM 19.2. The space $\mathscr{C}^{\infty}(\mathbb{X}, \mathbb{Y})$ is dense in $\mathscr{W}^{1, Q}(\mathbb{X}, \mathbb{Y})$.

Concerning integrability of the Jacobian determinant, the situation is dramatically different when $\mathbb{X}$ and $\mathbb{Y}$ are spheres [41]; just to illustrate:

EXAMPLE 19.3. Every Orlicz-Sobolev class $\mathscr{W}^{1, Q}\left(\mathbb{S}^{n}, \mathbb{S}^{n}\right)$ in which $Q(t)=o\left(t^{n}\right)$ contains an orientation preserving mapping whose Jacobian fails to be integrable.

This example rises several natural questions concerning function spaces of weakly differentiable mappings between given manifolds, for which the integral of the Jacobian represents the degree of a mapping.

Epilog. There are many more profound interactions between function spaces and nonlinear PDEs than we could possibly present here. The benefit is: wider applications of results and a greater understanding of the nature of nonlinear differential analysis. Let us just stress that the above advances were sparked by the early work of Władysław Orlicz. This is a testimony to his fantastic vision.

Spaces we need most are spaces we haven't discovered yet. 


\section{References}

[1] L. Ambrosio, N. Fusco and D. Pallara, Functions of Bounded Variation and Free Discontinuity Problems, Oxford Mathematical Monographs. The Clarendon Press, Oxford University Press, New York, 2000.

[2] R. J. Bagby, Maximal functions and rearrangements: some new proofs, Indiana Univ. Math. J. 32 (1983), 879-891.

[3] J. M. Ball, Convexity conditions and existence theorems in nonlinear elasticity, Arch. Rational Mech. Anal. 63 (1976/77), 337-403.

[4] J. M. Ball and R. D. James, Fine phase mixtures as minimizers of energy, Arch. Rational Mech. Anal. 100 (1987), 13-52.

[5] C. Bardaro, J. Musielak and G. Vinti, Nonlinear Integral Operators and Applications, de Gruyter Series in Nonlinear Analysis and Applications 9, de Gruyter, Berlin, 2003.

[6] C. Bennett and R. Sharpley, Interpolation of Operators, Pure and Applied Mathematics 129, Academic Press, Boston, MA, 1988.

[7] F. Bethuel, Approximation in Sobolev spaces between two manifolds and homotopy groups, in: Variational Methods, Progr. Nonlinear Differential Equations Appl. 4, Birkhäuser Boston, Boston, MA, 1990, 239-249.

[8] F. Bethuel, The approximation problem for Sobolev maps between two manifolds, Acta Math. 167 (1991), 153-206.

[9] L. Boccardo, Quelques problèmes de Dirichlet avec données dans de grands espaces de Sobolev, C. R. Acad. Sci. Paris Sér. I Math. 325 (1997), 1269-1272.

[10] A. Bonami, T. Iwaniec, P. Jones and M. Zinsmeister, On the product $B M O \cdot \mathscr{H}^{1}$, work in progress.

[11] J. Bourgain, Estimations de certaines fonctions maximales, C. R. Acad. Sci. Paris Sér. I Math. 301 (1985), 499-502.

[12] J. Bourgain, H. Brezis and P. Mironescu, Lifting in Sobolev spaces, J. Anal. Math. 80 (2000), 37-86.

[13] H. Brezis, N. Fusco and C. Sbordone, Integrability for the Jacobian of orientation preserving mappings, J. Funct. Anal. 115 (1993), 425-431.

[14] H. Brezis, Y. Li, P. Mironescu and L. Nirenberg, Degree and Sobolev spaces, Topol. Methods Nonlinear Anal. 13 (1999), 181-190.

[15] L. Carbone and R. De Arcangelis, Unbounded Functionals in the Calculus of Variations, Chapman \& Hall/CRC Monographs and Surveys in Pure and Applied Mathematics 125, Chapman \& Hall/CRC, Boca Raton, FL, 2002.

[16] M. Carozza and C. Sbordone, The distance to $L^{\infty}$ in some function spaces and applications, Differential Integral Equations 10 (1997), 599-607.

[17] R. Coifman, P.-L. Lions, Y. Meyer and S. Semmes, Compensated compactness and Hardy spaces, J. Math. Pures Appl. (9) 72 (1993), 247-286.

[18] R. R. Coifman and R. Rochberg, Another characterization of BMO, Proc. Amer. Math. Soc. 79 (1980), 249-254.

[19] R. R. Coifman, R. Rochberg and G. Weiss, Factorization theorems for Hardy spaces in several variables, Ann. of Math. (2) 103 (1976), 611-635.

[20] B. Dacorogna, Direct Methods in the Calculus of Variations, Applied Mathematical Sciences 78, Springer-Verlag, Berlin, 1989.

[21] B. Dacorogna and P. Marcellini, Implicit Partial Differential Equations, Progress in Nonlinear Differential Equations and their Applications 37, Birkhäuser Boston, Boston, MA, 1999. 
[22] S. K. Donaldson and D. P. Sullivan, Quasiconformal 4-manifolds, Acta Math. 163 (1989), 181-252.

[23] C. Fefferman, Characterizations of bounded mean oscillation, Bull. Amer. Math. Soc. 77 (1971), 587-588.

[24] A. Fiorenza, Duality and reflexivity in grand Lebesgue spaces, Collect. Math. 51 (2000), 131-148.

[25] A. Fiorenza and F. Giannetti, Regularity results for vector fields of bounded distortion and applications, JIPAM J. Inequal. Pure Appl. Math. 1 (2000), Article 14, 11 pp. (electronic).

[26] A. Fiorenza and G. Karadzhov, Grand and small Lebesgue spaces and their analogs, to appear.

[27] A. Fiorenza and J. M. Rakotoson, New properties of small Lebesgue spaces and their applications, Math. Ann. 326 (2003), 543-561.

[28] A. Fiorenza and C. Sbordone, Existence and uniqueness results for solutions of nonlinear equations with right hand side in $L^{1}$, Studia Math. 127 (1998), 223-231.

[29] N. Fusco and C. Sbordone, Higher integrability of the gradient of minimizers of functionals with nonstandard growth conditions, Comm. Pure Appl. Math. 43 (1990), 673-683.

[30] F. W. Gehring, The $L^{p}$-integrability of the partial derivatives of a quasiconformal mapping, Acta Math. 130 (1973), 265-277.

[31] F. Giannetti, T. Iwaniec, J. Onninen and A. Verde, Estimates of Jacobians by subdeterminants, J. Geom. Anal. 12 (2002), 223-254.

[32] F. Giannetti and A. Verde, Variational integrals for elliptic complexes, Studia Math. 140 (2000), 79-98.

[33] F. Giannetti and A. Verde, Lower semicontinuity of a class of multiple integrals below the growth exponent, Ann. Fac. Sci. Toulouse Math. (6) 10 (2001), 299-311.

[34] M. Giaquinta, Multiple Integrals in the Calculus of Variations and Nonlinear Elliptic Systems, Annals of Mathematics Studies 105, Princeton University Press, Princeton, NJ, 1983.

[35] E. Giusti, Metodi diretti nel calcolo delle variazioni, Unione Matematica Italiana, Bologna, 1994.

[36] L. Greco and T. Iwaniec, New inequalities for the Jacobian, Ann. Inst. H. Poincaré Anal. Non Linéaire 11 (1994), 17-35.

[37] L. Greco, T. Iwaniec and G. Moscariello, Limits of the improved integrability of the volume forms, Indiana Univ. Math. J. 44 (1995), 305-339.

[38] L. Greco, T. Iwaniec and C. Sbordone, Inverting the p-harmonic operator, Manuscripta Math. 92 (1997), 249-258.

[39] J. Gustavsson and J. Peetre, Interpolation of Orlicz spaces, Studia Math. 60 (1977), 33-59.

[40] P. Hajłasz, Equivalent statement of the Poincaré conjecture, Ann. Mat. Pura Appl. (4) (1994), 25-31.

[41] P. Hajłasz, T. Iwaniec, J. Malý and J. Onninen, Weakly differentiable mappings between manifolds, preprint.

[42] F. Hang and F. H. Lin, Topology of Sobolev mappings, preprint, 2001.

[43] T. Iwaniec, Unpublished notes.

[44] T. Iwaniec, P. Koskela and J. Onninen, Mappings of finite distortion: monotonicity and continuity, Invent. Math. 144 (2001), 507-531.

[45] T. Iwaniec and G. Martin, Geometric Function Theory and Non-linear Analysis, Oxford Mathematical Monographs, The Clarendon Press, Oxford University Press, New York, 2001 . 
[46] T. Iwaniec, L. Migliaccio, G. Moscariello and A. Passarelli di Napoli, A priori estimates for nonlinear elliptic complexes, Adv. Differential Equations 8 (2003), 513-546.

[47] T. Iwaniec and J. Onninen, $\mathscr{H}^{1}$-estimates of Jacobians by subdeterminants, Math. Ann. 324 (2002), 341-358.

[48] T. Iwaniec and C. Sbordone, On the integrability of the Jacobian under minimal hypotheses, Arch. Rational Mech. Anal. 119 (1992), 129-143.

[49] T. Iwaniec and C. Sbordone, Weak minima of variational integrals, J. Reine Angew. Math. 454 (1994), 143-161.

[50] T. Iwaniec and C. Sbordone, Quasiharmonic fields, Ann. Inst. H. Poincaré Anal. Non Linéaire 18 (2001), 519-572.

[51] T. Iwaniec and A. Verde, On the operator $\mathscr{L}(f)=f \log |f|$, J. Funct. Anal. 169 (1999), 391-420.

[52] T. Iwaniec and A. Verde, A study of Jacobians in Hardy-Orlicz spaces, Proc. Roy. Soc. Edinburgh Sect. A 129 (1999), 539-570.

[53] F. John and L. Nirenberg, On functions of bounded mean oscillation, Comm. Pure Appl. Math. 14 (1961), 415-426.

[54] N. Kalton and E. Saab (eds.), Banach Spaces (Berlin, 1985), Lecture Notes in Mathematics 1166, Springer-Verlag.

[55] R. A. Kerman and A. Torchinsky, Integral inequalities with weights for the Hardy maximal function, Studia Math. 71 (1981/82), 277-284.

[56] V. Kokilashvili and M. Krbec, Weighted Inequalities in Lorentz and Orlicz Spaces, World Sci., River Edge, NJ, 1991.

[57] M. A. Krasnosel'skiı̌ and J. B. Rutickiǐ, Convex Functions and Orlicz Spaces, translated from the first Russian edition by Leo F. Boron, Noordhoff, Groningen, 1961.

[58] M. Milman and R. Rochberg, The role of cancellation in interpolation theory, in: Harmonic Analysis and Operator Theory (Caracas, 1994), Contemp. Math. 189, Amer. Math. Soc., Providence, RI, 1995, 403-419.

[59] G. Moscariello, On the integrability of the Jacobian in Orlicz spaces, Math. Japon. 40 (1994), 323-329.

[60] S. Müller, Higher integrability of determinants and weak convergence in $L^{1}$, J. Reine Angew. Math. 412 (1990), 20-34.

[61] S. Müller, Variational models for microstructure and phase transitions, in: Calculus of Variations and Geometric Evolution Problems (Cetraro, 1996), Lecture Notes in Math. 1713, Springer, Berlin, 1999, 85-210.

[62] S. Müller, T. Qi and B. S. Yan, On a new class of elastic deformations not allowing for cavitation, Ann. Inst. H. Poincaré Anal. Non Linéaire 11 (1994), 217-243.

[63] J. Musielak, Orlicz Spaces and Modular Spaces, Lecture Notes in Mathematics 1034, Springer-Verlag, Berlin, 1983.

[64] W. Orlicz, Collected Papers, Part I, II, PWN-Polish Scientific Publishers, Warsaw, 1988. With contributions by Wanda Matuszewska and Lech Maligranda.

[65] M. M. Rao and Z. D. Ren, Theory of Orlicz Spaces, Monographs and Textbooks in Pure and Applied Mathematics 146, Marcel Dekker, New York, 1991.

[66] Y. G. Reshetnyak, Space Mappings with Bounded Distortion, Translations of Mathematical Monographs 73, Amer. Math. Soc., Providence, RI, 1989. Translated from the Russian by H. H. McFaden.

[67] R. Rochberg and G. Weiss, Analytic families of Banach spaces and some of their uses, in: Recent Progress in Fourier Analysis (El Escorial, 1983), North-Holland Math. Stud. 111, North-Holland, Amsterdam, 1985, 173-201. 
[68] R. Schoen and K. Uhlenbeck, Boundary regularity and the Dirichlet problem for harmonic maps, J. Differential Geom. 18 (1983), 253-268.

[69] E. M. Stein, Note on the class L $\log L$, Studia Math. 32 (1969), 305-310.

[70] E. M. Stein, Maximal functions. I. Spherical means, Proc. Nat. Acad. Sci. U.S.A. 73 (1976), 2174-2175.

[71] E. M. Stein, Harmonic Analysis: Real-Variable Methods, Orthogonality, and Oscillatory Integrals, Princeton Mathematical Series 43, Princeton University Press, Princeton, NJ, 1993.

[72] B. Stroffolini, On some integral inequalities, Rend. Accad. Sci. Fis. Mat. Napoli (4) 56 (1989), 29-39 (1990).

[73] J.-O. Strömberg and A. Torchinsky, Weighted Hardy Spaces, Lecture Notes in Mathematics 1381, Springer-Verlag, Berlin, 1989.

[74] A. Torchinsky, Real-Variable Methods in Harmonic Analysis, Pure and Applied Mathematics 123, Academic Press, Orlando, FL, 1986.

[75] H. Triebel, Interpolation Theory, Function Spaces, Differential Operators, second ed., Johann Ambrosius Barth, Heidelberg, 1995.

[76] H. C. Wente, An existence theorem for surfaces of constant mean curvature, J. Math. Anal. Appl. 26 (1969), 318-344.

[77] B. White, Homotopy classes in Sobolev spaces and the existence of energy minimizing maps, Acta Math. 160 (1988), 1-17. 